\title{
Bipolar Jets, Hot Interaction Regions, and Colliding Winds in OB Interacting Binaries
}

\author{
Geraldine J. Peters $\dagger$ \\ Space Sciences Center \& Department of Physics \& Astronomy, University of Southern \\ California, Los Angeles, CA 90089-1341. \\ email: gjpeters@mucen.usc.edu
}

\begin{abstract}
Contemporary information on the nature of the circumstellar environment in earlytype interacting binaries derived from observation is discussed. Emphasis is placed on results from FUV spectroscopy. New spectra from the FUSE spacecraft and earlier FUV observations with $I U E$ have revealed the presence of hot interaction regions, bipolar outflows, splash outflows, and accretion hot spots in Algol-type systems with B-type mass gainers. In addition, close Otype pairs and systems containing $\mathrm{O}+\mathrm{W}$ stars show a shock-heated region generated from their colliding winds. Recent FUSE observations of an apparent hot accretion spot and associated splash plasma in the direct-impact system U Cephei are presented.
\end{abstract}

Keywords. binaries: close, circumstellar matter, stars: early-type, ultraviolet: stars

\section{Introduction}

It has been more than 30 years since the publication of the landmark papers on mass flow in semidetached binary stars by Lubow and Shu $(1975,1976)$. FUV spectroscopy from the IUE, ORFEUS-SPAS II, HST, and FUSE spacecrafts and Doppler tomography using optical data have provided observational confirmation of many structures discussed in the Lubow and Shu papers and revealed other components of the circumstellar (CS) material that were not predicted from their gas dynamical calculations. In this paper we discuss some of the key results from FUV observations. Information derived from tomography is presented by Richards in another article contained in these proceedings.

The behavior of the mass accretion in Algol binaries can be understood with the aid of the $r-q$ diagram in which the fractional radius of the mass gainer $\left(\mathrm{R}_{\mathrm{p}} / \mathrm{a}\right)$ is plotted versus the mass ratio, $\mathrm{q}=\mathrm{M}_{\text {loser }} / \mathrm{M}_{\text {gainer }}$, and compared with the computations of gas stream hydrodynamics by Lubow \& Shu (1975). A representative $r-q$ is shown in Figure 1. The upper dashed curve, $\varpi_{\mathrm{d}}$, delineates the fractional radius of the dense accretion disk for systems of different mass ratios. The lower dashed curve, $\varpi_{\min }$, shows the minimum distance that a stream particle will achieve relative to the center of the gainer. If a system falls above the upper curve, the gas stream will strike the photosphere of the mass gainer and if any $\mathrm{H} \alpha$ emission from CS material is seen, it will be transient. If the system falls below the lower curve, one will see a prominent accretion disk that emits strongly in the Balmer lines and other species. The most variable accretion disks are found in systems that fall between the two curves, since the inner part of the wide gas stream will strike or graze the photosphere of the primary while the outer edge will feed an accretion disk. These concepts underlie the discussion below.

$\dagger$ Based on observations made with the NASA-CNES-CSA Far Ultraviolet Spectroscopic Explorer. FUSE is operated for NASA by the Johns Hopkins University under NASA contract NAS5-32985. 


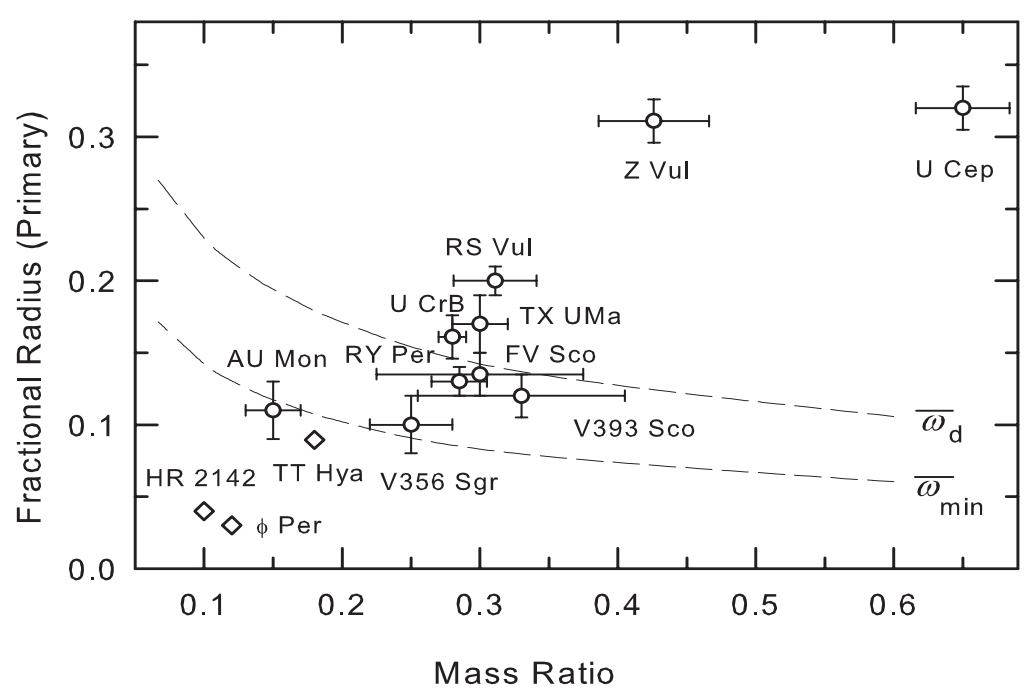

Figure 1. The locations of several well-known Algol systems in the $r-q$ diagram. The diamonds show systems with prominent disks.

\section{Hot Interaction Regions}

Evidence for a high temperature plasma above the trailing hemisphere in an Algol system was first reported by Plavec (1983). IUE observations of the direct-impact system $\mathrm{U}$ Cep during the total phase of its primary eclipse revealed the presence of prominent emission lines from highly-ionized species such as N V, C IV, and Si IV that imply the presence of a CS plasma with a temperature of $\sim 100,000 \mathrm{~K}$. The strongest component of the emission was mapped to CS gas on the side of the binary that sustained the gas stream impact. About the same time Peters \& Polidan (1984, PP84) found absorption lines from the latter species in the phase interval 0.5-0.9 in IUE spectra of AU Mon, CX Dra, and U CrB. The latter authors called the CS plasma in which these absorption lines are formed the High Temperature Accretion Region (HTAR). Both Plavec and PP84 suggested that the plasma is heated from a shock, or a series of shocks, produced by the impacting gas stream and PP84 concluded that the HTAR features are formed by resonance scattering in a plasma of high temperature $\left(T_{\text {ion }} \sim 10^{5} \mathrm{~K}\right)$, intermediate density $\left(\mathrm{N}_{\mathrm{e}} \sim 10^{9} \mathrm{~cm}^{-3}\right)$, and moderate-high carbon depletion. IUE data acquired later in the mission revealed that the HTAR prevails in the systems in the upper two-thirds of the $r-q$ diagram where stable disks cannot form and the azimuthal extent of the HTAR depends on the period of the binary (Peters 2001).

\section{Bipolar Flows}

Although IUE spectra provided considerable information on the nature of the CS material in the orbital plane in Algol binaries (Peters 2001), it has been the FUSE spacecraft (Moos et al. 2000) that has revealed the prevalence of a highly-ionized plasma flow above/below the plane. Prior to FUSE, research teams found evidence for jet-like structures in the disk system $\beta$ Lyr. Combining optical interferometry, spectroscopy, and photometry a consortium of researchers in the mid-1990s concluded that most of the $\mathrm{H} \alpha$ and He II emission in $\beta$ Lyr is formed in bipolar jets (Harmanec et al. 1996). This conclusion was reinforced by Hoffman et al. (1998) who combined optical and FUV 


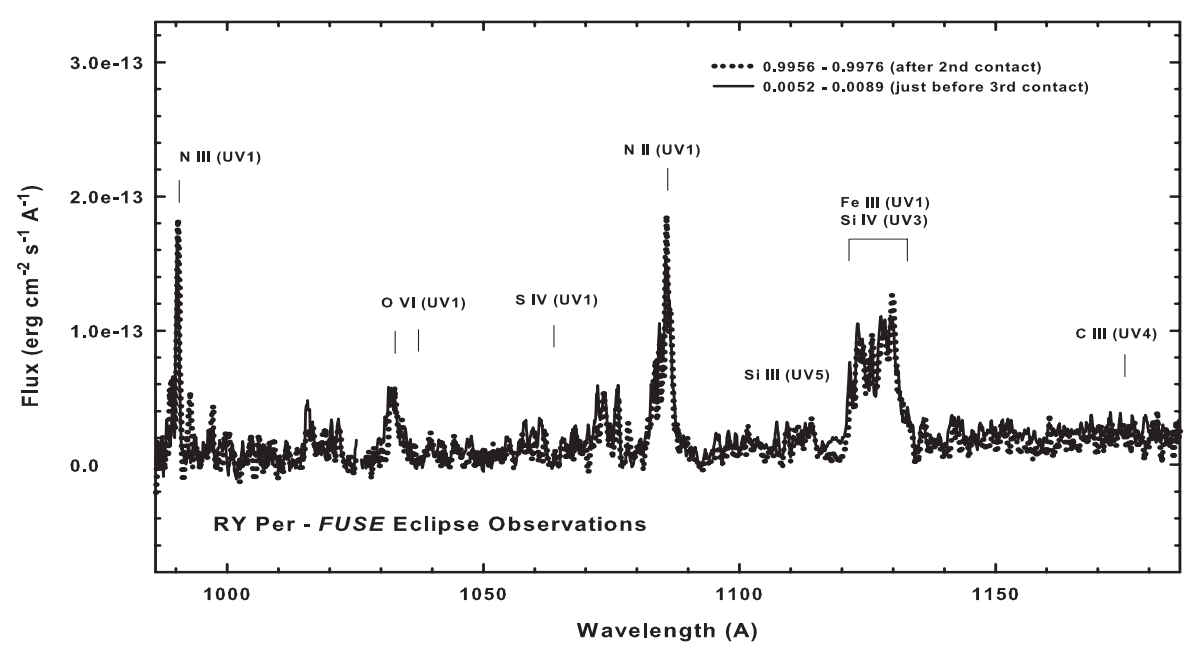

Figure 2. FUSE observations of RY Per taken during totality on 2002 December 8 reveal the presence of emission lines from highly-ionized species and evidence for extreme CNO processing in the material that is currently being transferred.

spectropolarimetry (from the WUPPE instrument flown on the ASTRO 2 shuttle mission) to produce a model where the line formation region for the Balmer emission, UV emission lines, and NUV continuum is oriented perpendicular to the orbital plane where the visible continuum originates.

FUV spectra from FUSE have recently been used to map the location of the hot CS plasma in three Algol binaries (cf. Peters \& Polidan 2004). Phase-resolved spectra in the region $\lambda \lambda 950-1190 \AA$ of $\mathrm{V} 356 \mathrm{Sgr}\left(\mathrm{B} 3 \mathrm{~V}+\mathrm{A} 2 \mathrm{II}, \mathrm{P}=8.90^{\mathrm{d}}\right)$, TT Hya $(\mathrm{B} 9.5 \mathrm{Ve}$ + K1III-IV, 6.95 $)$, and RY Per (B4V + F7III-IV, 6.86 ${ }^{\mathrm{d}}$ ) obtained during the total eclipse $\dagger$ have revealed the presence of optically-thin, broad (FWHM $\sim 600-1200 \mathrm{~km} \mathrm{~s}^{-1}$ ) emission lines from $\mathrm{O}$ VI in all three systems. O VI $\lambda 1032 \AA$ is the dominant feature in the FUSE spectrum of V356 Sgr. These features suggest the presence of a 300,000 K component to the CS plasma. Since only slight variations are observed in the O VI lines throughout totality, we have concluded that the emission originates in a turbulent outflow perpendicular to the orbital plane. In contrast, the emission from moderate-ionization species such at C III, N II, and Si III tends to be double-peaked and displays a type of $\mathrm{V} / \mathrm{R}$ variability that suggests the features are formed in a disk (cf. Peters 2001, Peters \& Polidan 2004).

The FUSE totality observations of RY Per are especially noteworthy (Figure 2). The level of ionization appears to be slightly higher in this system than in the other two program objects. O VI and the lines from the moderately-ionized species display a FWHM of about $800 \mathrm{~km} \mathrm{~s}^{-1}$ and very little change between phase 0.997 and 0.007 . All of the emission appears to be originating in a bipolar outflow. There is evidence for extreme CNO-processing in the CS plasma that forms the emission features. Emission from the resonance lines of $\mathrm{N}$ II, III is especially strong and there is no emission from C III $\lambda 977$ and $1176 \AA$ that is observed in V356 Sgr and TT Hya. From an analysis of IUE LORES spectra of RY Per taken during totality, Olson \& Plavec (1997) also concluded that the

$\dagger$ The emission lines can be seen only during totality in Algol systems in the upper two-thirds portion in the $r-q$ diagram. Emission is visible at all phases in the prominent disk systems. 


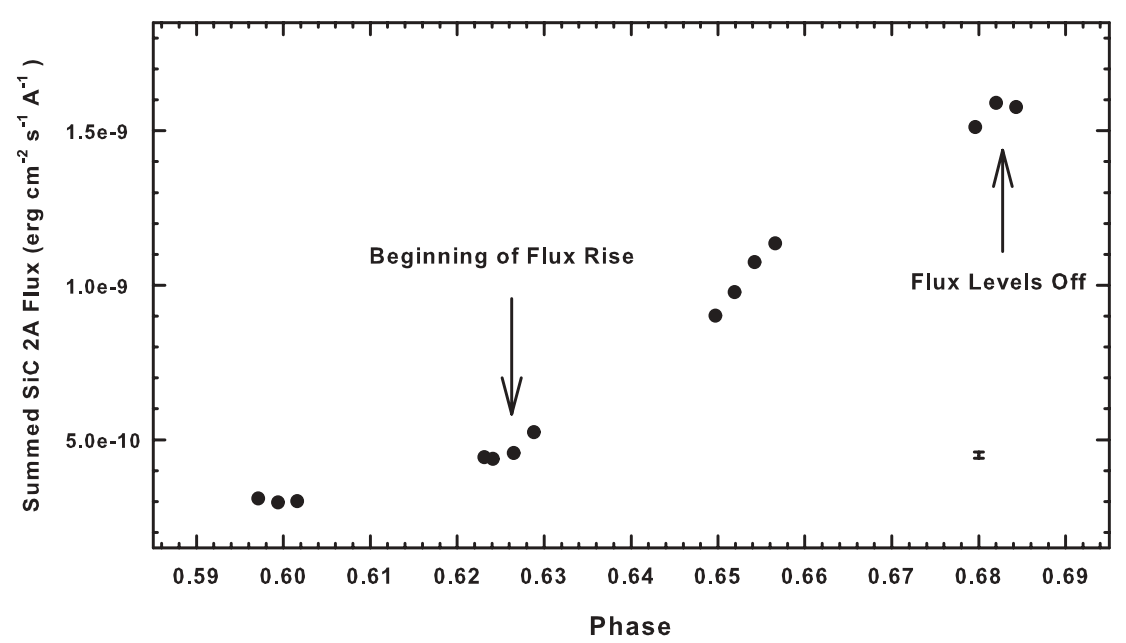

Figure 3. Summed flux from the FUSE $\mathrm{SiC} 2 \mathrm{~A}$ detector for each $8^{\mathrm{m}}$ exposure obtained during the long 2004 February 09 observation plotted versus phase. Note that an apparent accretion hot spot rotates into our line-of-sight at phase 0.625 and is fully visible at phase 0.682 . A typical error bar (lower right) is about the width of the plotted points.

CS plasma in which the emission lines are formed is N-rich and C-poor. But the spectral resolution of the $I U E$ data was too low to obtain information on the velocity behavior of the lines. Since photospheric absorption from C III $1176 \AA$ is clearly seen in RY Per, we conclude that the material in the bipolar outflow originates in the gas steam from the mass-losing star and has had only minimal interaction with the photospheric material.

\section{Hot Accretion Spots and Splash Regions}

In Peters (2001) I identified a splash plasma as one of the components of the CS material in Algol-type interacting binaries. This region is detected from the presence of violet-shifted absorption lines from moderate to high-ionization species and is located downstream from the impact site. The material appears to have been reflected from the mass gainer's photosphere and heated by the shock from the encounter. The splash plasma is mostly seen in systems in the middle portion of the $r-q$ diagram (e.g., RY Per, FV Sco, V393 Sco) but as we will see from the FUSE observations of U Cep presented below, it can also reside above the impact site of a short-period system.

$\mathrm{U}$ Cep $\left(\mathrm{B} 7 \mathrm{~V}+\mathrm{G} 8 \mathrm{III}-\mathrm{IV} ; \mathrm{P}=2.49^{\mathrm{d}}\right)$ has been observed with the FUSE spacecraft at random phases since 2002 as part of the FUSE Survey and Supplemental Program. During the course of a five hour observation on 2004 February 9, we observed a rather spectacular rise in the level of the FUV flux that is shown in Figure 3. We have plotted the sum of the flux recorded by the $\mathrm{SiC} 2 \mathrm{~A}$ detector for each of the 14 exposures versus phase. The flux begins to rise at phase 0.625 and levels off by phase 0.682 . We interpret this photometric behavior as evidence for a "hot" accretion spot that rotated into our line-ofsight. The spot would be centered at about phase 0.90 , very close to the azimuth where we would expect the gas stream to impact photosphere of the B star. From the time it took for the spot to fully come into view, we can place a lower limit of $20^{\circ}$ on its size. The spot would occupy $\sim 2 \%$ of the facing hemisphere of the B star. The FUV flux level observed in a FUSE observation taken at a phase of 0.28 on the following day, 2004 February 10, 


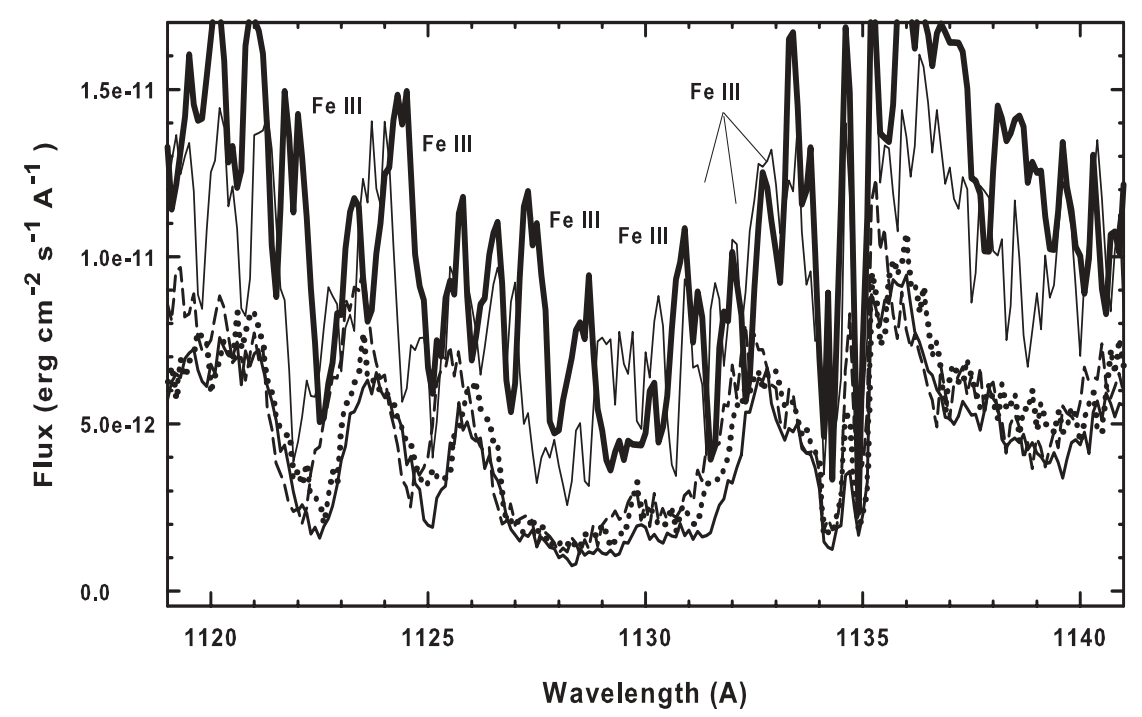

Figure 4. Selected exposures from the FUSE LiF 2a/Lif 1b observations of U Cep of 2004 February $9 \& 10$ compared with a spectrum taken on 2002 September 1 at a phase of 0.83 (bold line). Exposure 16 on February 9 obtained at a phase of 0.68 at flux maximum is plotted with a thin line. The spectra showing a lower flux level are Exposures 2 (phase 0.60, medium solid line) and 7 (phase 0.63, dotted line, just before flux rise) on February 9, and Exposure 1 on February 10 (phase 0.28 , dashed line). The locations of Fe III shell-type lines seen in the 2002 spectrum are indicated.

was comparable to the pre-rise value. An elevated flux was observed on two other dates (2002 September 1, phase 0.83, and 2003 February 13, phase 0.12). At these phases the hot spot would be located to the left (later phase) of our line-of-sight, and on the receding limb, respectively. A lower flux level was observed in six other observations taken between phases $0.22-0.58$ (spot behind the facing hemisphere). Obvious "shell-type" features of Fe III and similar species, that are redshifted by $\sim 150 \mathrm{~km} \mathrm{~s}^{-1}$ relative to the B star, were observed at phase 0.83 (cf. Figure 4 ). Weaker, blueshifted $\left(\sim-200 \mathrm{~km} \mathrm{~s}^{-1}\right)$ sharp lines were seen on 2004 February 9 (post-rise), but there was no discernible shell structure at phase 0.12. The brightest FUV flux was observed at phase 0.83. The plasma in which these features are formed appears to be limited in angular extent and has a temperature in the range 20,000-40,000 K (based upon the collisional ionization calculations of Shull \& Van Steenberg 1982) that is 2-3 times higher than the photosphere of the mass gainer. The Fe III plasma is most certainly associated with the impact site. The component observed above the impact region is clearly associated with the mass infall, but the material downstream appears to have been splashed from the impact site.

\section{Colliding Winds}

In close $\mathrm{O}$ star pairs and $\mathrm{O}+\mathrm{W}$ binaries, a collision is inevitable between their massive, fast-moving $\left(\mathrm{V}_{\infty} \sim-1000-4000 \mathrm{~km} \mathrm{~s}^{-1}\right.$ ) winds. Plasma heating occurs along a bow shock that is curved around the star with the weaker wind (St-Louis et al. 2006). A shock cone produces $\mathrm{P}$ Cygni features of C III, IV, O VI, Si IV, S IV, and P V. St-Louis et al. (2005) have recently modeled the bow shock region in Sanduleak 1 (W04+O4) in the SMC from FUSE data. Iping et al. (2006, poster presentation at this meeting) are currently analyzing new FUSE observations of five other massive O star pairs with short 
periods $\left(2.2-15.1^{\mathrm{d}}\right)$ in the our Galaxy and the Magellanic Clouds that will ultimately reveal further information on the interaction zone.

\section{Concluding Remarks}

During the past two decades FUV spectroscopy has provided a wealth of information on OB interacting binaries. Collisions between a gas stream and the mass gainer's photosphere or stellar winds produce a shock-heated plasma that radiates in spectral lines from highly-ionized species that can only be observed in the FUV. Bipolar jets may be commonplace in Algol-type systems. Evidence for a hot bipolar outflow has been found in all three Algol binaries that have been observed during totality with the FUSE spacecraft. From polarimetry Piirola et al. (2005) concluded that a high-latitude spot or flow exists in the disk system W Ser. Evidence for a high velocity polar stream has recently been found in the direct-impact system U CrB from a 3-D Doppler tomography analysis of $\mathrm{H} \alpha$ data (Agafonov et al. 2006). The relationship between the bipolar flow, splash plasma, and the HTAR is presently unknown but all of these regions appear be formed by the impacting gas stream. The plasmas reside around or downstream from the impact site. The bipolar flow could simply be splashed material that is shock-heated then injected into the CS environment. The observations of U Cep described above support this idea. The possible role of a magnetic field that originates in the late-type mass loser and becomes "frozen-in" to the gas stream has yet to be investigated. Information on the CS environment in OB interacting binaries will continue to improve with future FUV observations and 3-D magnetohydrodynamic simulations of mass flow.

\section{Acknowledgements}

The analysis of the FUSE observations on U Cep is a collaborative effort with B-G Andersson, T. Ake, and R. Sankrit, whom I would like to thank for fruitful discussions. The author appreciates support from NASA grants NAG5-12253 and NNG04GL17G.

\section{References}

Agafonov, M., Richards, M.T., \& Sharova, O. 2006, ApJ 652, 1547

Harmanec, P. et al. 1996, A\& A 312, 879

Hoffman, J.L., Nordsieck, K.H., \& Fox, G.K. 1998, AJ 115, 1576

Lubow, S.H. \& Shu, F.H. 1975, ApJ 198, 383

Lubow, S.H. \& Shu, F.H. 1976, ApJ 207, L53

Moos, H.W. et al. 2000, ApJ 538, L1

Olson, E.C. \& Plavec, M.J. 1997, AJ 113, 425

Peters, G.J. 2001 in: D. Vanbeveren (ed.), The Influence of Binaries on Stellar Population Studies (Kluwer), p. 79

Peters, G.J. \& Polidan, R.S. 1984, ApJ 283, 745 (PP84)

Peters, G.J. \& Polidan, R.S. 2004, Astron. Nachr. 325, 225

Piirola, V., Berdyugin, A., Mikkola, S., \& Coyne, G.V. 2005, ApJ 632, 576

Plavec, M.J. 1983, ApJ 275, 251

Shull, J.M. \& Van Steenberg, M. 1982, ApJS 48, 95

St-Louis, N., Moffat, A.F.J., Marchenko, S., \& Pittard, J.M. 2005, ApJ 628, 953

St-Louis, N., Moffat, A.F.J., Marchenko, S., Pittard, J.M., \& Boisvert, P. 2006, in: G. Sonneborn, Moos, H. W., \& B.-G. Andersson (eds.), Astrophysics in the Far Ultraviolet, Five Years of Discovery with FUSE (ASP Conference Series, Vol. 348), p. 121 